\title{
Estrategias de manejo de Senecio vulgaris L. asociado al cultivo de Daucus carota L. a partir de los principios de la modelación biológica
}

\section{Management strategies of Senecio vulgaris L. associated to Daucus carota $L$. from the principles of biological modeling}

\author{
Mónica Yadira Dotor-Robayo'; Ana Cruz Morillo-Coronado²
}

\begin{abstract}
1Ing. Agrónomo, M.Sc., Ph.D. (c). Universidad Nacional de Colombia, Ciencias Agrarias. Bogotá, Colombia; e-mail: mydotorr@unal.edu.co; (DD http://orcid. org/0000-0002-2133-0450

${ }^{2}$ Ing. Agrónoma, Ph.D. Universidad Pedagógica y Tecnológica de Colombia - UPTC, Tunja - Boyacá, Colombia; e-mail: ana.morillo@uptc.edu.co; D http:// orcid.org/0000-0003-3125-0697
\end{abstract}

Cómo citar: Dotor-Robayo, M.Y.; Morillo-Coronado, A.C. 2020. Estrategias de manejo de Senecio vulgaris L. asociado al cultivo de Daucus carota L. a partir de los principios de la modelación biológica. Rev. U.D.C.A Act. \& Div. Cient. 23(1):e1058. http://doi. org/10.31910/rudca.v23.n1.2020.1058

Artículo de acceso abierto publicado por Revista U.D.C.A Actualidad \& Divulgación Científica, bajo una licencia Creative Commons CC BY-NC 4.0

Publicación oficial de la Universidad de Ciencias Aplicadas y Ambientales U.D.C.A, Institución de Educación Superior Acreditada de Alta Calidad por el Ministerio de Educación Nacional.

Recibido: Noviembre 20 de 2019

Aceptado Mayo 19 de 2020

Editado por: Ingeborg Zenner de Polanía

\section{RESUMEN}

Senecio vulgaris L. es una maleza, considerada de difícil control cuando se asocia a la zanahoria, debido a que compite por recursos con el cultivo, siendo importante su control, ya que tiene un impacto negativo en la productividad. La modelación biológica es una herramienta que permite prever los posibles efectos del control de la especie, ya que identifica las diferentes variables de un sistema y los factores que las afecta, para de este modo, predecir los resultados en diversos ambientes. Con base en la dinámica de sistemas y los aspectos morfo-fisiológicos de la especie, se planteó la modelación del banco de semillas de $S$. vulgaris, basado en las ecuaciones planteadas por Fernández-Quintanilla, donde se construyó el diagrama Forrester, al que se le incorporaron las diferentes ecuaciones y valores del modelo, para proyectar la dinámica de la especie en el banco de semillas, dependiendo del manejo. Se propusieron tres escenarios: (1) control con herbicidas en post-emergencia, (2) rotación de cultivos y (3) incorporar los métodos culturales de control, con un testigo en pre siembra y rotación de cultivos. Los resultados del modelo muestran que cuando se realiza rotación de cultivos y se incorporan manejos en pre-siembra al proceso convencional de la especie, se presenta una menor cantidad de semillas en el banco.

Palabras clave: dinámica de sistemas; diagrama Forrester; modelos biológicos; malezas; banco de semillas.

\section{ABSTRACT}

Senecio vulgaris L. is a weed, considered difficult to control, when it is associated with carrot, because it competes for resources with the crop, its control being important, since it has a negative impact on 
crop productivity. Biological modeling is a tool that allows predicting the possible effects of the species control, since it identifies the different variables of a system and the factors that affect them, in order to predict the results in different environments. Based on the dynamics of systems and the morphophysiological aspects of the species, the modeling of the S. vulgaris seed bank was suggested, based on the equations proposed by Fernández-Quintanilla, the Forrester diagram was constructed, which was incorporated the different equations and values of the model, to project the dynamics of the species in the seed bank, depending on the management. Three scenarios were proposed, (1) post-emergence herbicide control, (2) crop rotation (3) incorporate traditional control methods with a control in presowing and crop rotation. The model results show that when crop rotation is performed and pre-sowing management is incorporated into the conventional process of the species, a smaller quantity of seeds is presented in the bank.

Keywords: system dynamics; Forrester diagram; biological models; weeds; seed bank.

\section{INTRODUCCIÓN}

El sector de Tierra Negra, en el municipio de Ventaquemada, departamento de Boyacá, Colombia, es reconocido como una importante región productora de papa y hortalizas (AGRONET, 2019); en estos sistemas productivos es frecuente encontrar plantas que le hacen competencia, como kikuyo (Cenchrus clandestinus), gualola (Poligonium nepalense) y cerrajilla (Senecio vulgaris), que se controlan con aplicaciones de herbicidas, como cletodim o fluazifop-p butyl (hoja angosta), metribuzina y linuron (hoja ancha), solos o en mezcla (Kim et al. 2018). Es común que la aplicación de estos herbicidas se haga en post-emergencia y sin tener en cuenta las consideraciones técnicas correspondientes.

En los últimos años, en los cultivos de zanahoria de Ventaquemada, se ha encontrado, con mayor frecuencia, la maleza $S$. vulgaris, especie considerada de difícil control y alta capacidad competitiva e invasiva (Zhu et al. 2017), por lo cual, es una de las especies más dominantes, asociada a los sistemas productivos de la región. Lo anterior, debido a las prácticas de control de malezas que se han implementado en los cultivos, el momento de exposición al herbicida y metabolismo de la especie, entre otros, permitiendo a la especie, dependiendo de la fase fenológica, tener escapes a los herbicidas aplicados (Dotor et al. 2018).

A la fecha son escasos los estudios acerca de la dinámica poblacional de $S$. vulgaris, que es una especie de origen mediterráneo anual, con inflorescencia compuesta, que se reproduce por semillas y, para la zona expresa un potencial reproductivo de hasta 16.000 semillas por planta, estructuras reproductivas, que exhiben viabilidades superiores al 90\%, es sensible a la competencia inter e intraespecífica, la cual, se refleja en la producción de semilla y biomasa, reduciendo la productividad de los cultivos (Dotor \& Cabezas, 2015; Cheng et al. 2019).

La modelación biológica, se puede abordar desde la dinámica de las poblaciones, que componen el sistema objeto de estudio; sin embargo, para que las predicciones arrojadas por los modelos, sean lo más precisas a la realidad, es necesario que la información que alimenta el modelo sea lo más ajustada a lo que se registra en campo (Chay et al. 2012). Los resultados proyectados a partir del modelo deben permitir un alto nivel de precisión, que contribuya en la toma de decisiones, sobre las acciones a ejecutar, en cualquier sistema productivo (Schrieber et al. 2016).

Existen diferentes modelos establecidos para evaluar el efecto de una especie sobre otra, por ejemplo, el modelo depredadorpresa de Lotka Volterra (Anisiu, 2014) o el modelo general de manejo de malezas de Wiles et al. (1996), que aportan información, que permiten plantear un modelamiento poblacional y, a la vez, muestran que en la diversidad de modelos, la selección de uno u otro y el planteamiento de las ecuaciones que lo componen, son fundamentales para la validez del escenario proyectado (Chay et al. 2012).

Teniendo en cuenta lo anterior, González \& Quintanilla (2004) evaluaron la dinámica de poblaciones, considerando como punto de referencia su demografía, las características propias de los ciclos de vida de cada especie y los estados fenológicos, para llegar a determinar las características del banco de semillas, como también, los parámetros que se requieren para alimentar un modelo y establecer la proyección del comportamiento futuro del escenario que se desea conocer.

En este proceso es fundamental la estructuración del diagrama Forrester, que permite la implementación de un modelo dinámico, a través del establecimiento de las variables de nivel, de flujo y auxiliares del sistema (Ma et al. 2012).

Con base en este proceso y partiendo de los resultados obtenidos en diferentes ensayos en campo, esta investigación tuvo como objetivo principal generar un modelo biológico, que proyectara la dinámica del banco de semillas de $S$. vulgaris, en el municipio de Ventaquemada, departamento de Boyacá y que permitiera prever el efecto de diferentes opciones de manejo sobre este banco de semillas en los cultivos de Daucus carota, para así contribuir a la sostenibilidad y rentabilidad del cultivo.

\section{MATERIALES Y MÉTODOS}

La construcción del modelo dinámico, se estableció en diferentes etapas: en la primera, se seleccionó el modelo de dinámica de poblaciones de González \& Quintanilla (2004), utilizado para Lolium rigidum; posteriormente, se identificaron las variables dinámicas, que intervienen en el sistema Senecio-Zanahoria; los valores base establecidos para alimentar el modelo fueron determinados a partir del seguimiento fenológico de la especie, en competencia intra e inter específica, obteniendo los valores correspondientes al número de inflorescencias por planta, número de semillas por planta, producción de semillas en condiciones libres de competencia, implementando las metodologías planteadas por Dotor \& Cabezas (2015). 
El porcentaje de germinación, se estableció considerando el total de las semillas de una inflorescencia, que se llevaron a cajas de Petrí, a $6{ }^{\circ} \mathrm{C}$ y en exposición a luz blanca, durante 20 días, en donde cada caja representó una unidad experimental, para un total de 100 unidades experimentales.

La sobrevivencia de las plántulas, se calculó mediante conteos de germinación, luego de sembrar 50 semillas en $30 \mathrm{~cm}^{2}$ de suelo; la incidencia de factores abióticos, se determinó teniendo en cuenta el control que ejerció el herbicida sobre las 100 semillas en el suelo. Ésta, se realizó mediante pruebas en invernadero y suelo, utilizando un número variable de semillas por $\mathrm{m}^{2} \mathrm{y}$ determinando el efecto de la densidad de plantas, sobre la germinación, crecimiento, desarrollo, fecundidad y sobrevivencia. Los datos para alimentar el modelo, se tomaron en el momento de la germinación y, luego, en intervalos de 8 días. Todas las variables consideradas en el estudio, ya habían sido anteriormente validadas en otras investigaciones (Dotor \& Cabezas, 2015; Dotor et al. 2018).

Una vez establecidos los datos obtenidos, a través de las evaluaciones realizadas en campo, se procedió a la construcción del diagrama Forrester y a la incorporación de las ecuaciones en el modelo, utilizando el programa Vensim ${ }^{\circledR}$ PLE Versión 6.2(X32).

En el diagrama Forrester, se incorporaron las ecuaciones de simulación (González \& Quintanilla, 2004), en donde cada una de las variables, estuvo alimentada por variables auxiliares o valores constantes, que corresponden a los datos que se alimenta el modelo y que, en este caso, provienen de los resultados obtenidos en los ensayos de conocimiento de las especies.

En la figura 1, se puede observar el diagrama de Forrester, donde se determinaron como constantes del modelo el control de plántulas, las plántulas sobrevivientes, el área requerida para el desarrollo de las plántulas, el número de semillas producidas por planta, cuando crecen sin competencia, los controles ejercidos por los procesos de competencia interespecifica y por los herbicidas, utilizados en el cultivo y la proporción de semillas perdidas en el banco, por factores bióticos y abióticos.

Estas constantes, se asociaron a las variables auxiliares de semillas $\mathrm{m}^{-2}$, plantas maduras, fecundidad, producción de semillas y pérdidas en el banco; como variable de flujo, fue definido el ingreso en el banco de semillas y la variable de nivel y el banco de semillas (Figura 1). Para el proceso de modelación, se partió de un banco de semillas, con una densidad de 10 semillas $\mathrm{m}^{-2}$.

Ecuaciones del modelo. Las ecuaciones consideradas para el desarrollo del modelo biológico fueron las adoptadas por González \& Quintanilla (2004):

Fecundidad. Determinada como $\mathrm{F}=\mathrm{fs} *(1-\mathrm{Lc})(1-\mathrm{Wc} 2)(1-\mathrm{Tc} 3) /$ $(1+\mathrm{aMt})$

En donde, F: Fecundidad; fs: Número de semillas producidas por una planta sin competencia; a: Área requerida para producir el

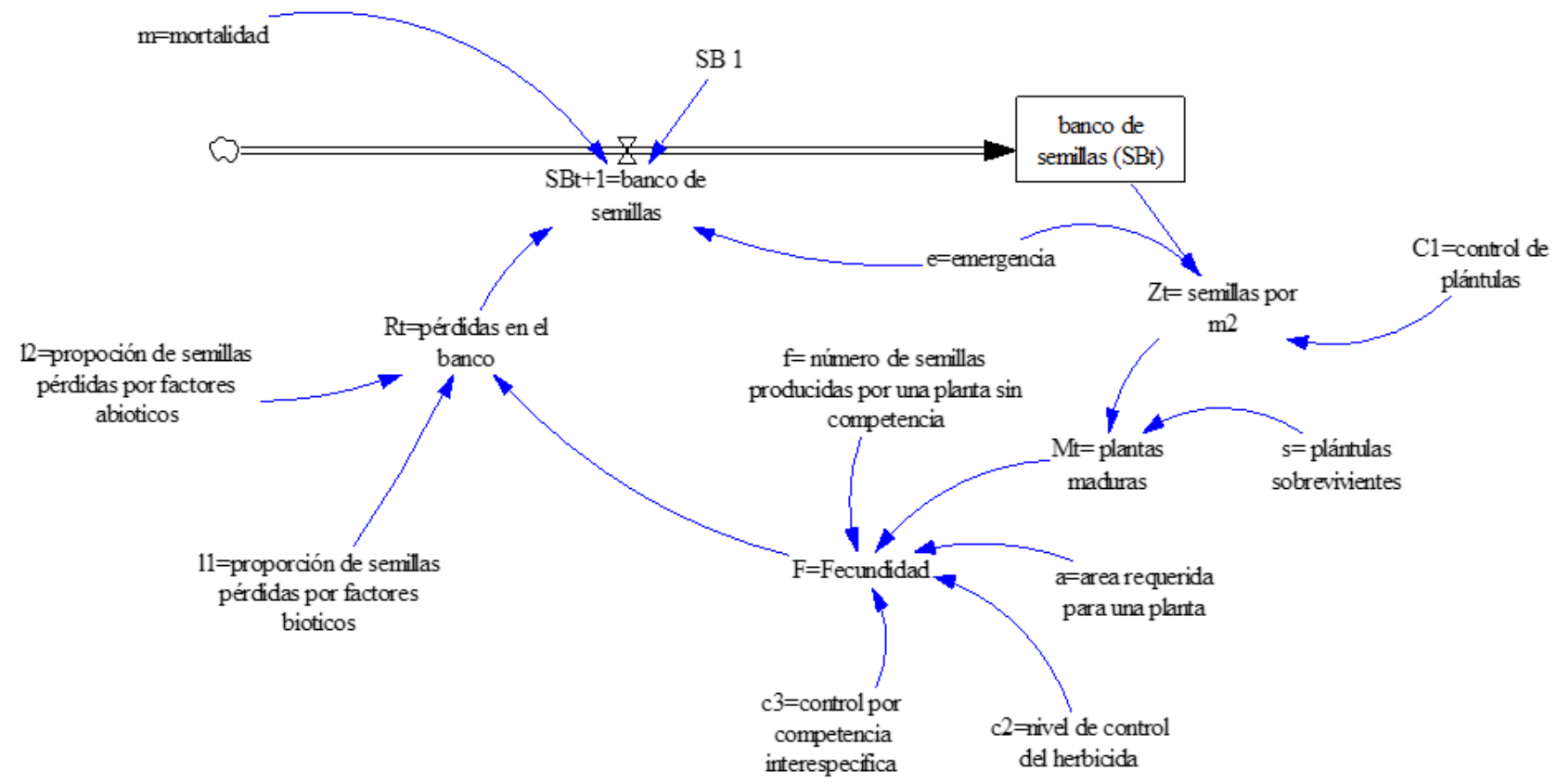

Figura 1. Diagrama de Forrester, que incluye las variables comprendidas en la construcción del modelo $\mathrm{m}^{2}$. 
número $\mathrm{f}$ de semillas; Mt: Proporción de plantas sobrevivientes hasta la madurez; Lc: Afectación en la producción de semillas por competencia con el cultivo por luz; Wc: Afectación en la producción de semillas por competencia con el cultivo por agua; Tc: Afectación en la producción de semillas por competencia con el cultivo; fs: Número de semillas producidas por la planta sin competencia.

Pérdidas en el banco. Determinada como Rt: F (1-Af)*(1-Bf)

En donde, Rt: Semillas después de pérdidas; F: Fecundidad; Af: Factores abióticos; Bf: Factores bióticos.

Tamaño del banco de semillas para el siguiente año. Determinado por la ecuación: SBt+1: SBt (1-e) (1-m)+Rt.

En donde, SBt: Emergencia en el banco en un año (t); m: Proporción del banco que muere; e: Proporción del banco que emerge.

Población inicial del banco de semillas. Determinada por la ecuación: Sbt: $\int \mathrm{SBt}+1$.

\section{Emergencia de plántulas Zt. (1-C1)eSBt}

Donde, C1: Población controlada con los métodos tradicionales (afecta la producción de semillas); e: Emergencia proporcional en el banco de semillas; SBt: Población inicial del banco de semillas.

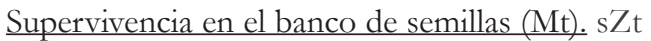

Donde, Mt: Plantas maduras; s: Plántulas sobrevivientes.

Los aspectos correspondientes a control por competencia interespecífica con el cultivo, la proporción de pérdidas, debida a factores bióticos, se reportan en la tabla 1.

Se plantearon tres escenarios para el banco de semillas de $S$. vulgaris, teniendo en cuenta los resultados obtenidos en la evaluación de esta especie bajo las condiciones agroclimáticas de la zona en estudio (Dotor \& Cabezas, 2015; Cardenal et al. 2016; Dotor et al. 2018), que se pueden observar en detalle en el cuadro 1.

Escenario 1. Control en pre-emergencia. Se evaluó el efecto de la aplicación de herbicidas sobre el banco de semillas de la maleza, considerando tres niveles: a) medio, (b) bajo, (c) alto y el testigo (d), en libre crecimiento sin control o manejo alguno, sobre el cual, se supondría el uso de un herbicida pre-emergente; en los niveles a y b, herbicida selectivo a la maleza, aplicado en post-emergencia, lo que implica un porcentaje medio y alto de plantas, que escapan al control del herbicida, debido a que han salido de la ventana de control que ejerce el mismo y (c), aplicación de un herbicida selectivo, en preemergencia y post-emergencia temprana, que ejerce un control alto de la maleza (Tabla 1 y Cuadro 1).

Cuadro 1. Escenarios y modelos relacionados.

\begin{tabular}{|c|c|}
\hline Escenario & Descripción \\
\hline \multirow{4}{*}{$\begin{array}{l}\text { Escenario } 1 . \\
\text { control con } \\
\text { herbicidas }\end{array}$} & (a) bajo pre y pos emergente usado en pos-emergencia tardía control estimado de $40 \%$ \\
\hline & (b) medio pre y pos emergente selectivo usado en pos-emergencia temprana control estimado de $60 \%$ \\
\hline & (c) alto de herbicida en pre y pos emergente en preemergencia control estimado de $80 \%$ \\
\hline & (d) plantas en libre crecimiento, sin aplicación de herbicida control estimado de $0 \%$ \\
\hline \multirow{2}{*}{$\begin{array}{l}\text { Escenario } 2 . \\
\text { competencia inter- } \\
\text { especifica }\end{array}$} & (e) Competencia inter-especifica con el cultivo de zanahoria sin manejo adicional \\
\hline & (f) Competencia inter-especifica con el cultivo de pasturas \\
\hline \multirow{3}{*}{$\begin{array}{l}\text { Escenario } 3 \text {. } \\
\text { rotación y manejo } \\
\text { integrado }\end{array}$} & $\begin{array}{l}\text { (g) Competencia inter-especifica con el cultivo de zanahoria más manejo químico en preemergencia y } \\
\text { post-emergencia }\end{array}$ \\
\hline & (h) Competencia inter-especifica con el cultivo de pasturas más manejo químico \\
\hline & $\begin{array}{l}\text { (i) Competencia inter-especifica con el cultivo de zanahoria en condiciones de manejo tradicional } \\
\text { (d) plantas en libre crecimiento, sin aplicación de herbicida control estimado de } 0 \%\end{array}$ \\
\hline
\end{tabular}


Tabla 1. Parámetros utilizados para construir el modelo.

\begin{tabular}{|c|c|c|}
\hline Parámetros & Nomenclatura & $\begin{array}{l}\text { Parámetro en el } \\
\text { modelo }\end{array}$ \\
\hline Emergencia & $\mathrm{E}$ & 0,654 \\
\hline Plántulas sobrevivientes (Daucus carota) & s1 & $0,1 / 0,9$ \\
\hline Plántulas sobrevivientes (Cenchrus clandestinus) & s2 & 0,1 \\
\hline Fecundidad & $\mathrm{F}$ & 13890 \\
\hline Semillas pérdidas por cosecha & 11 & 0,23 \\
\hline Semillas pérdidas por captura & 12 & 0,58 \\
\hline Mortalidad en el banco de semillas & M & 0,95 \\
\hline Control de plántulas (retrasado) & c1 & 0,6 \\
\hline $\begin{array}{l}\text { Control con herbicida con efecto sobre el } 80 \% \text { de la población } \\
\text { (aplicación de herbicida en preemergencia y post-emergencia temprana) }\end{array}$ & c2 & 0,8 \\
\hline $\begin{array}{l}\text { Control con herbicida con efecto sobre el } 60 \% \text { de la población } \\
\text { (aplicación de herbicida en preemergencia y post-emergencia) }\end{array}$ & c2 & 0,6 \\
\hline $\begin{array}{l}\text { Control con herbicida con efecto sobre el } 40 \% \text { de la población } \\
\text { (aplicación de herbicida en post emergencia) }\end{array}$ & c2 & 0,4 \\
\hline Efecto por competencia con el cultivo & c3 & 0,11 \\
\hline Banco de semillas & $\mathrm{SBt}$ & 8.286 \\
\hline Área requerida para una planta sin competencia & A & 0,32 \\
\hline
\end{tabular}

Escenario 2. Cultivo sin aplicación de herbicida. Se observa la dinámica del banco de semillas de $S$. vulgaris, cuando este crece (e), en competencia intraespecífica con el cultivo de zanahoria (baja habilidad competitiva), sin aplicar herbicidas y (f), cuando crece en competencia interespecífica con pasturas (alta habilidad competitiva), igualmente, sin aplicación de herbicidas (Tabla 1 y Cuadro 1). En los dos cultivos, se plantea un manejo adicional, en el cual, se simula la utilización previa de herbicida en pre-emergencia, para el control de las poblaciones acompañantes.

Escenario 3. Manejo integrado. Se modeló simulando tres posibilidades de manejo: en la primera, se implementó un cultivo de zanahoria -escenario de competencia intraespecífica $(\mathrm{g})$, con efecto en el desarrollo fenológico de la especie (Dotor \& Cabezas, 2015)-, en el cual, se mantuvieron las condiciones tradicionales de la zona, que consiste en la utilización de herbicidas, previendo un control poblacional del 70\% (aplicación de herbicidas selectivos en preemergencia y post-emergencia temprana p.e. linuron o metribuzina); la segunda, hace referencia a la población $S$. vulgaris creciendo en competencia total con pasturas (h), junto con la aplicación de herbicidas $y$, finalmente, la tercera, que se obtiene realizando una rotación de cultivos, entre zanahoria y pasturas, incorporando la aplicación de herbicidas en pre-siembra para ambos cultivos y en post-emergencia, para el caso de la zanahoria.

Todos los escenarios fueron contrastados con relación al manejo tradicional del cultivo que, frecuentemente, consiste en el control de la maleza en post-emergencia al cultivo, con el uso habitual de metribuzina y una posterior aplicación de linuron en dosis comercial, aplicaciones que habitualmente se realizan entre 1.5 y 2 meses después de la emergencia del cultivo (Tabla 1), lo que genera escapes de aquellas plantas de malezas, que han superado el umbral de acción del herbicida.

\section{RESULTADOS Y DISCUSIÓN}

En las evaluaciones realizadas en este estudio, se encontraron los siguientes resultados por escenarios:

Escenario 1. Control en pre-emergencia. En esta proyección, que se evaluó el manejo con herbicidas y los tres posibles comportamientos del banco, basados en la probabilidad de escape de las plantas al control del herbicida, se observó que, en la primera proyección, se logró el control del 80\% de las poblaciones emergidas de S. vulgaris; en la segunda, el $60 \%$ y, en la tercera, el $40 \%$. Resultados similares a los obtenidos en las investigaciones realizadas en garbanzo y yuca, que se demuestra la efectividad del uso de herbicidas en dosis intermedias y estados tempranos de desarrollo del cultivo (Soltero-Díaz et al. 2010).

En todos los casos, el modelo registra, en los primeros años, un poblamiento rápido por parte de las semillas de la especie; según los datos modelados, la presencia de la maleza, junto con su alta capacidad de producción de semilla, hacen que, durante el primer año, se genere una alta infestación y, en los años posteriores, tiende a mantenerse constante, estabilidad que refleja la densidad máxima 
de semillas, mediada por el manejo modelado, la dinámica de la especie y el control químico seleccionado (Délye et al. 2016; Dotor et al. 2018).

En este tipo de manejo, el modelo del banco de semillas muestra una ecuación exponencial (Tabla 2) que, en todos los casos, presenta un valor de $\mathrm{R}^{2}$ mayor a 0,8 . Producto del modelamiento, se ajustó la curva al modelo $\mathrm{F}=\mathrm{a}^{*} \mathrm{x}^{\mathrm{b}}$; con $(\mathrm{x}>0)$, cuyas constantes, se pueden observar en la tabla 2.

En el escenario de mayor control de la población (uso de herbicidas post-emergentes), se obtendría 858 semillas $\mathrm{m}^{-2}$ al término del año uno; 1.045 , en el año dos y esta cantidad continua en aumento, de modo que, en el año 10, al término de la simulación, se tiene una cantidad de 1.997 semillas $\mathrm{m}^{-2}$ en el banco. Este escenario, se repite en su tendencia, pero con mayores cantidades de semillas en los demás tratamientos y proyecta cómo, a corto plazo, se presenta la mayor infestación de semillas en el banco, en donde luego del primer año, este crecimiento presenta un comportamiento moderado (Cardenal et al. 2016).

La disminución en los porcentajes de control de la especie genera infestaciones en el banco de semillas, que son porcentualmente mayores, de modo que, al término de la modelación, en el banco donde se obtiene un control del 80\%, la infestación es de 1.997 semillas $\mathrm{m}^{2}$; el control del $60 \%$, presenta 3.040 semillas $\mathrm{m}^{2}$; el control del 40\%, tendría 3.950 semillas $\mathrm{m}^{2}$ y el testigo, 5.621 semillas $\mathrm{m}^{2}$ (Figura 2a, Tabla 2). Por lo anterior, se plantea la necesidad de un manejo adecuado y oportuno de las malezas, dependiendo de la especie y las condiciones agroclimatológicas del sitio (Cardenal et al. 2016).

Escenario 2. Cultivo sin aplicación de herbicida. En la segunda proyección, cuando se considera la dinámica del banco de semillas de S. vulgaris, bajo dos condiciones de competencia, se pudo observar que con las pasturas, que presentan una alta habilidad competitiva (Alonso-Ayuso et al. 2018), se generó una disminución significativa de semilla de la maleza en el banco (Tabla 2), llegando a valores máximos de 1.807 semillas $\mathrm{m}^{-2}$, número significativamente menor que la cantidad producida, cuando la planta está en proceso de competencia con el cultivo, en donde los valores arrojados del modelo indican una infestación del banco, con alrededor de 5.553 semillas $\mathrm{m}^{-2}$ (Figura 2b).

La competencia intraespecífica es uno de los procesos fundamentales, que modifica el crecimiento de una planta dentro de una población, mientras que la interespecífica afecta diferentes especies (Autran et al. 2013). La estructura y productividad, se encuentra fuertemente influenciada por las relaciones competitivas entre sus componentes (González \& Quintanilla, 2004); esto es válido, tanto para los sistemas naturales como para los agroecosistemas. La competencia, se puede definir como una interacción entre individuos, provocada por la demanda común de un recurso limitado y que conduce a la reducción en la supervivencia, crecimiento o reproducción de, al menos, uno de los competidores (Storkey \& Neve, 2018). Es un fenómeno complejo, definido por múltiples factores, como se puedo evidenciar en los resultados encontrados y en la evaluación poblacional de diferentes malezas (Schrieber et al. 2016; Young, 2018).

Escenario 3. Manejo integrado. El modelamiento de estos escenarios, se observa en la figura $2 c$, donde se puede observar el comportamiento de la maleza cuando se integran estrategias de manejo diferentes a la aplicación de herbicidas, aprovechando los procesos de competencia que se producen entre especies (Dotor et al. 2018; Lemay et al. 2018).

Se pudo observar, que cuando se mantienen las prácticas tradicionales, se obtiene la mayor cantidad de semillas de $S$. vulgaris en el banco, por lo que el mantener esta condición sin ninguna variación -incluida

Tabla 2. Ecuación resultante de la modelación, desglosada por escenario planteado.

\begin{tabular}{|l|c|c|c|}
\hline \multicolumn{1}{|c|}{ Escenario } & a & b & $\mathbf{R}^{2}$ \\
\hline (a) Control 40\% & $2247,73 \pm 47,69$ & $0,228 \pm 0,012$ & 0,98 \\
\hline (b) Control 60\% & $1526,64 \pm 33,8$ & $0,284 \pm 0,012$ & 0,986 \\
\hline (c) Control 80\% & $815,86 \pm 15,6$ & $0,379 \pm 0,01$ & 0,99 \\
\hline (d) Plantas en libre crecimiento (sin control) & $3705,5 \pm 66,19$ & $0,164 \pm 0,01$ & 0,985 \\
\hline (e) Competencia con zanahoria sin manejo tradicional & $3705,56 \pm 66,15$ & $0,164 \pm 0,010$ & 0,98 \\
\hline (f) Competencia con Pasturas sin manejo adicional & $704,01 \pm 12,48$ & $0,40 \pm 0,009$ & 0,99 \\
\hline $\begin{array}{l}\text { (g) Competencia inter-especifica con Pasturas + manejo con } \\
\text { herbicidas }\end{array}$ & $270,21 \pm 1,19$ & $0,524 \pm 0,002$ & 0,99 \\
\hline (h) Rotación pasturas - zanahoria & $351,88 \pm 145,13$ & $0,135 \pm 0,2$ & 0,09 \\
\hline
\end{tabular}

Términos de la ecuación ajustada al modelo $\mathrm{F}=\mathrm{a}^{*} \mathrm{x}^{\mathrm{b}}$ en cada uno de los escenarios evaluados. Donde, a y b= constantes de la ecuación generadas por la condición evaluada; $\mathrm{X}=$ año; $\mathrm{R}^{2}=$ valor del ajuste de la ecuación al modelo $\mathrm{F}=$ Efecto del control sobre la población con $\mathrm{X}>0$ o tamaño del banco de semillas para la condición evaluada. 

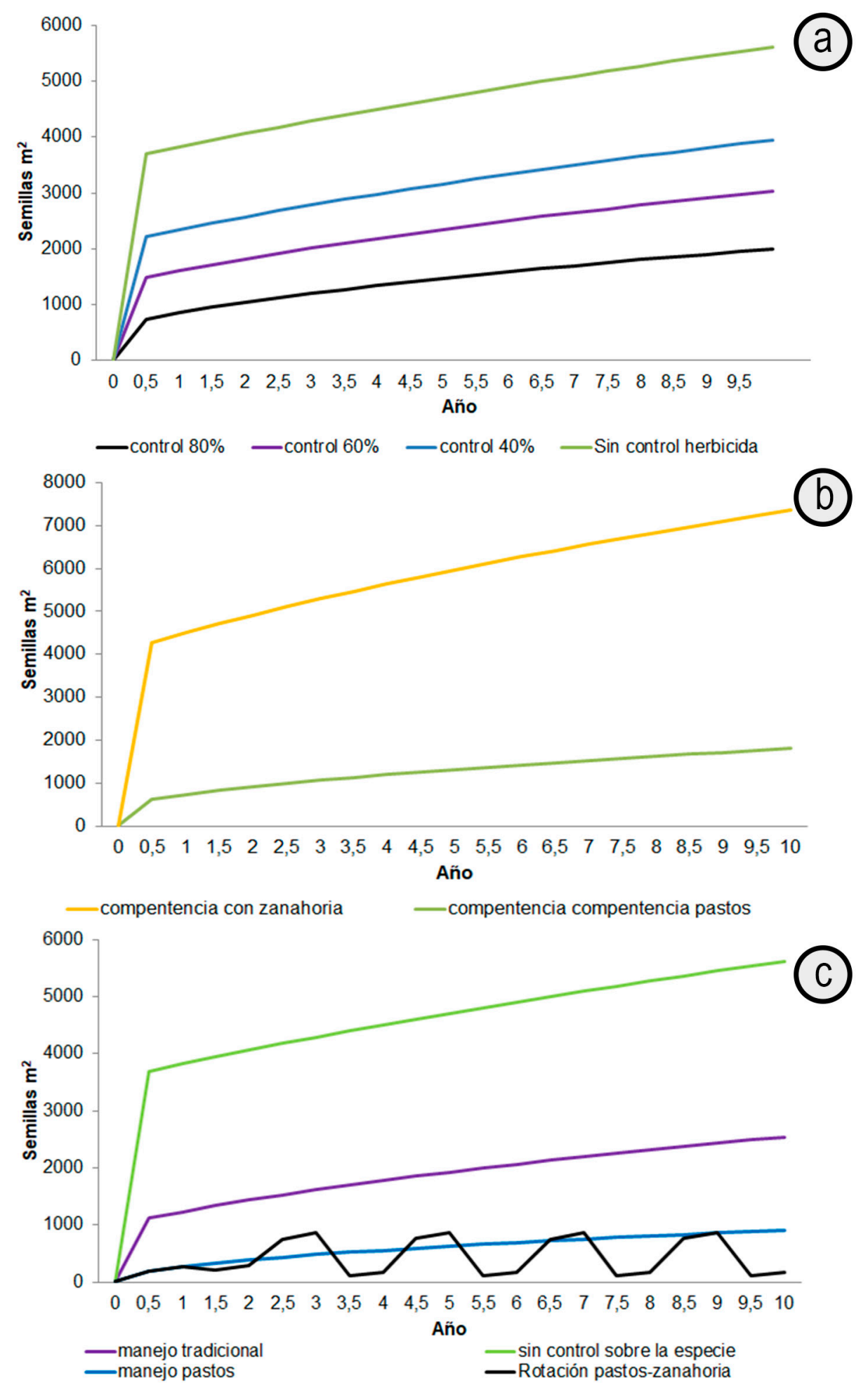

Figura 2. Modelación según cada uno de los escenarios: a. proyección del Banco de semillas de S. vulgaris con la aplicación de herbicidas, en cuatro escenarios-control; b. proyección del Banco de semillas de $S$. vulgaris en competencia interespecífica, con siembra de pasturas y con zanahoria; c. proyección del Banco de semillas de $S$. vulgaris cuando se implementa un modelo de manejo integrado de la población de S. vulgaris. 
la rotación del herbicida-, traerá como consecuencia una mayor dominancia de la especie, proceso que estaría mediado por el uso exclusivo de herbicidas, para el control de una población, en donde se debe tener en cuenta el momento de aplicación del herbicida y el hecho de que la maleza no es sensible a los herbicidas, durante todo su estado fisiológico, lo que genera una mayor cantidad de semilla en el banco (Dotor \& Cabezas, 2015; Cardenal et al. 2016; Dotor et al. 2018).

Este escenario muestra que el uso de herbicidas no garantiza la sostenibilidad del sistema y potencializa el ingreso de variables no contempladas, como es el de la resistencia a herbicidas, presionada por la selección de poblaciones que se genera, por lo cual, es necesario el planteamiento de estrategias sostenibles para el control eficiente de las malezas, en cualquier sistema productivo (Dotor \& Cabezas, 2015; Young, 2018).

Como se había observado anteriormente, en este escenario, se encontró también, que cuando hay competencia interespecífica con los pastos, el banco de semillas mostró resultados significativamente menores a los observados con un cultivo de menor habilidad competitiva, como es $D$. carota. Por lo anterior, el modelamiento dependerá de la especie y la interacción con las otras presentes en el sistema productivo, lo cual, pone de manifiesto la necesidad de conocer las diferentes interacciones entre las diversas especies que habitan dentro de un mismo agroecosistema (Autran et al. 2013; Storkey \& Neve, 2018).

Cuando se implementa en este escenario la rotación de cultivos, se pudo observar que el tamaño del banco de semillas era oscilante, mostrando la dependencia del mismo, debida al cultivo establecido y la viabilidad de las semillas en el banco, ya que, en el caso de los pastos, la reducción en la cantidad de semillas va a estar influenciada por la competencia del cultivo y la perturbación en el suelo, como consecuencia de la rotación de cultivos y por factores bióticos y abióticos, propios del sistema agrícola, hecho que ya ha sido reportado por otros investigadores (Hosseini et al. 2014).

Cabe mencionar, que todas las variables evaluadas en este estudio, ya habían sido validadas en estudios previos de dinámica poblacional de $S$. vulgaris, por lo cual, validan su uso en estudios tendientes a determinar su comportamiento dentro de un agroecosistema. De las simulaciones proyectadas, se puede inferir que, en el caso de la rotación de cultivos, si bien se observa oscilante en el proceso (Figura 2c, Tabla 2), también permite una mayor sostenibilidad, ya que la variación en el flujo de semillas al banco, generan retroalimentación positiva y negativa, que permite estabilidad y que el sistema se mantenga en el tiempo. Resultados similares fueron reportados por Werle et al. (2016), quienes enfatizan en la importancia de la rotación de cultivos y su influencia en las dinámicas poblacionales de las malezas.

El escenario de menor cantidad de semillas en el banco de $S$. vulgaris, se observó al aumentar la competencia interespecífica, resultado que se sustenta en la plasticidad del cultivo y efecto de competencia sobre la especie (Cardenal et al. 2016; Dotor et al. 2018). Para el caso de $S$. vulgaris, diversos estudios han demostrado, que esta especie es sensible a la competencia inter e intraespecífica, observándose que cuando la planta se somete a procesos de competencia por agua, luz y nutrientes, genera unas modificaciones en su fisiología, que afectan la floración y el número de semillas por planta; de allí, que surja la competencia como un aspecto fundamental en el proceso de producción de semillas en la especie (Dotor \& Cabezas, 2015).

La dinámica de sistemas ofrece herramientas que permiten prever el comportamiento futuro e incorporarlas en el proceso de producción, va a ser importante para acercarse a los procesos de agricultura de precisión, en donde el manejo adecuado de los factores intervinientes permite disminuir las brechas agrícolas en producción, de modo que sea óptima y, por tanto, sostenible en el tiempo (Cheng et al. 2019).

Para esto, se debe introducir en el proceso la perspectiva holística, de modo que la actividad de producción, no solo se reduzca el sembrar-fumigar-abonar-recolectar, sino que el proceso incluya el manejo adecuado de los componentes del sistema, de modo que se puede prever y limite la aparición de poblaciones de difícil control o resistentes, como lo sugieren Beckie \& Tardif (2011) y, entre otros, Boer et al. (2019).

Los resultados encontrados en este estudio presentaron diferentes escenarios en la dinámica cultivo-maleza, que al analizarlos en sus diferentes dimensiones y modelarlos con las ecuaciones biológicas, se puede inferir, que es necesario continuar con los manejos tradicionales, que consideren la incorporación de estrategias más amigables con el ambiente, como la rotación de cultivos y no propender por el uso reiterativo de herbicidas, ciclo tras ciclo de producción en el cultivo de zanahoria, sin ninguna consideración técnica.

Agradecimientos: Al profesor Marco Cabezas Gutiérrez, por todo su apoyo y colaboración. Conflicto de intereses: El manuscrito fue preparado y revisado con la participación de todos los autores, quienes declaramos que no existe conflicto de intereses que ponga en riesgo la validez de los resultados encontrados.

\section{REFERENCIAS}

1. AGRONET. 2019. Red de información y comunicación del sector agropecuario. Colombia. Cifras agropecuarias Disponible desde Internet en: www.agronet.gov.co (con acceso 11/10/2020).

2. ALONSO-AYUSO, M.; GABRIEL, J.; GONZÁLEZ, I.; DEL MONTE, J.; QUEMADA, M. 2018. Weed density and diversity in a long-term cover crop experiment background. Crop Protection. 112(1):103-111. https:// doi.org/10.1016/j.cropro.2018.04.012

3. ANISIU, M.C. 2014. Lotka, Volterra and their model. Didáctica mathematica. 32(1):9-17.

4. AUTRAN, V.; PURICELLI, E.; FACCINI, D.; PALAZZESI, G.; BENITEZ, C. 2013. Competencia entre Adesmia bicolory distintas especies de malezas. Ciencias Agronómicas. 21:7-11. 
5. BECKIE, H.; TARDIF, F. 2011. Herbicide cross resistance in weeds. Crop Protection. 35:15-28. https://doi. org/10.1016/j.cropro.2011.12.018

6. BOER, T.; SMITH, P.; CHANDLER, K.; NURSE, R.; OBEID, K.; SWANTON, C. 2019. A linuron-free sede management strategy for carrots. Weed Technology, 33(3):464-474. https://doi.org/10.1017/wet.2018.112

7. CARDENAL, Z.; TORRES, D.; DOTOR, M.; MORILLO, A. 2016. Caracterización del banco activo de semillas en cultivos de zanahoria del municipio de Villa Pinzón (Cundinamarca). Rev. U.D.C.A Act. \& Div. Cient. 19(2):297306. https://doi.org/10.31910/rudca.v19.n2.2016.83

8. CHAY, D.; DELFÍN, H.; MELÉNDEZ, V.; GONZÁLEZ, A. 2012. Revisión de modelos predictivos de especies de control biológico. Tropical and Subtropical Agroecosystems. 15(1):337-356.

9. CHENG, D.; TIAN, Z.; FENG, L.; XU, L.; WANG, H. 2019. Diversity analysis of the rhizospheric and endophytic bacterial communities of Senecio vulgaris L. (Asteraceae) in an invasive range. Peer J Life \& Environment. 6(1):1-24. https://doi.org/10.7717/peerj.6162

10. DÉLYE, C.; CAUSSE, R.; SÉVERINE, M. 2016. Genetics basis, evolucionary origin and spread of resistance to herbicides inhibiting acetolactate synthase in common groundsel (Senecio vulgaris). Pest Management Science, 72(1):89-102. https://doi.org/10.1002/ps.4058

11. DOTOR, M.; CABEZAS, M. 2015. Determinación de la habilidad competitiva entre Daucus carota L. y Senecio vulgaris L. Rev. U.D.C.A Act. \& Div. Cient. 18(1):81-89. https:// doi.org/10.31910/rudca.v18.n1.2015.456

12. DOTOR, M.; GONZÁLEZ, L.; MORILLO, A. 2018. Período crítico de competencia de la zanahoria (Daucus carota L.) y malezas asociadas al cultivo. Rev. Ciencias Agrícolas. 35(1):5-15. https://doi.org/10.22267/rcia.183501.78

13. GONZÁLEZ, J.; QUINTANILLA, F. 2004. Modelling the population dynamics of annual ryegrass (Lolium rigidum) under various weed management systems. Crop Protection. 23(8):723-729. https://doi.org/10.1016/j. cropro.2003.12.007

14. HOSSEINI, P.; KARIMI, H.; BABAEI, S.; MASHHADI, H.; OVEISI, M. 2014. Weed seed bank as affected by crop rotation and disturbance. Crop Rotation. 64(1):1-6. https:// doi.org/10.1016/j.cropro.2014.05.022

15. KIM, J.; LEE, J.; LEE, I. 2018. Distribution, germination characteristics, and management of common groundsel
(Senecio vulgaris) in Korea. Weed \& Turfgrass Science. 7(4):309-320. https://doi.org/10.5660/WTS.2018.7.4.309

16. LEMAY, J.; TELFER, Z.; DUPREE, C.; McDONALD, M. 2018. The impact of the carrot rust fly and carrot weevil integrated pest management program on the grounddwelling beetle complex in commercial carrot fields at the Holland marsh, Ontario, Canada. Environmental Entomology. 47(4):788-794. https://doi.org/10.1093/ ee/nvy078

17. MA, C.; ZHANG, G.; ZHANG, X.; ZHOU, B.; MAO, T. 2012. Simulation modeling for wetland utilization and protection based on system dynamic model in a coastal city, China. Procedia Environmental Sciences. 13(2):202-213. https:// doi.org/10.1016/j.proenv.2012.01.019

18. SCHRIEBER, K.; WOLF, S.; WYPIOR, C.; HÖHLIG, D.; LACHMUTH, K. 2016. Adaptative and non-adaptative evolution of trait means and genetic trait correlations for herbivory resistance and performance in an invasive plant. Oikos. 126(4):572-582. https://doi.org/10.1111/oik.03781

19. SOLTERO-DIAZ, L.; PEREZ-DOMINGUEZ, J.; VALENCIA-BOTIN, A. 2010. Evaluación de herbicidas para el control de malezas en garbanzo (Cicer arietinum L.) de riego en la región Ciénaga de Chapala, México. Ciencia y tecnología agrícola. 19(2):85-88.

20. STORKEY, J.; NEVE, P. 2018. What good is weed diversity?. Weed Research. 58: 239-243.https://doi.org/10.1111/ wre.12310

21. WERlE, R.; TENHUMBERG, B.; LINDQUist, J. 2016. Modeling shatter cane dynamics in herbicidetolerant grain sorghum cropping systems. Ecological Modeling. 343(1):131-141. https://doi.org/10.1016/j. ecolmodel.2016.10.023

22. WILES, L.; KING, R.; SCHWEIZER, E.; LYBECKER, D.; SWINTON, S. 1996. GWM: General weed management model. Agricultural Systems. 50(4):355-376.

23. YOUNG, S.L. 2018. Beyond precision weed control: A model for true integration. Weed Technology. 32(1):7-10. https:// doi.org/10.1017/wet.2017.70

24. ZHU, B.; BARRET, S.; ZHANG, D.; LIAO, W. 2017. Invasion genetics of Senecio vulgaris: loss of genetic diversity characterizes the invasion of a selfing anual, despite multiple introductions. Biological Invasions. 19(1):255267. https://doi.org/10.1007/s10530-016-1277-0 